UCRL-PROC-221144

Applying Science and

Technology to Combat WMD Terrorism

C. R. Wuest, R. W. Werne, Billy W. Colston,

C. L. Harmann-Siantar

May 5, 2006

SPIE Defense and Security Symposium, Orlando, Florida, April 17-21, 2006 
This document was prepared as an account of work sponsored by an agency of the United States Government. Neither the United States Government nor the University of California nor any of their employees, makes any warranty, express or implied, or assumes any legal liability or responsibility for the accuracy, completeness, or usefulness of any information, apparatus, product, or process disclosed, or represents that its use would not infringe privately owned rights. Reference herein to any specific commercial product, process, or service by trade name, trademark, manufacturer, or otherwise, does not necessarily constitute or imply its endorsement, recommendation, or favoring by the United States Government or the University of California. The views and opinions of authors expressed herein do not necessarily state or reflect those of the United States Government or the University of California, and shall not be used for advertising or product endorsement purposes. 


\title{
Applying Science and Technology to Combat WMD Terrorism
}

\author{
Craig R. Wuest, Roger W. Werne, Billy W. Colston, and Christine L. Hartmann-Siantar \\ Lawrence Livermore National Laboratory \\ P.O. Box 808 \\ Livermore, CA 94550
}

\begin{abstract}
Lawrence Livermore National Laboratory (LLNL) is developing and fielding advanced strategies that dramatically improve the nation's capabilities to prevent, prepare for, detect, and respond to terrorist use of chemical, biological, radiological, nuclear, and explosive (CBRNE) weapons. The science, technology, and integrated systems we provide are informed by and developed with key partners and end users. LLNL's long-standing role as one of the two principle U.S. nuclear weapons design laboratories has led to significant resident expertise for health effects of exposure to radiation, radiation detection technologies, characterization of radioisotopes, and assessment and response capabilities for terrorist nuclear weapons use. This paper provides brief overviews of a number of technologies developed at LLNL that are being used to address national security needs to confront the growing threats of CBRNE terrorism.
\end{abstract}

Keywords: Chemical, Biological, Radiological, Nuclear, Explosive, Counterterrorism

\section{INTRODUCTION}

Lawrence Livermore National Laboratory (LLNL) is developing and fielding advanced strategies that dramatically improve the nation's capabilities to prevent, prepare for, detect, and respond to terrorist use of radiological, nuclear, chemical, or biological weapons. The science, technology, and integrated systems we provide are informed by and developed with key partners and end users. LLNL's long-standing role as one of two principle nuclear weapons design laboratories has led to significant resident expertise for health effects of exposure to radiation, radiation detection technologies, characterization of radioisotopes, and assessment and response capabilities for terrorist nuclear weapons use. A catalyst for LLNL's focus on civilian chemical and biological counterterrorism was the 1995 nerve gas attack in Tokyo's subway. LLNL began the Chemical and Biological National Security Program (CBNP) the same year using internal, LaboratoryDirected Research and Development investments with a focus on biodetection. The Nunn-Lugar-Domenici Defense Against Weapons of Mass Destruction Act, passed in 1996, initiated several U.S. nonproliferation and counterterrorism programs, including the DOE's Chemical and Biological Nonproliferation Program. In 2003, the Department of Homeland Security (DHS) was formed, and most of the DOE program was transferred to the DHS. Today, LLNL's programs in Chemical, Biological, Radiological, Nuclear, and Explosive (CBRNE) counterterrorism, shown schematically in Figure 1, are sponsored by the DOE, National Nuclear Security Administration, DHS, National Institutes of Health, U.S. Department of Agriculture, and several other defense, intelligence, and law enforcement organizations. ${ }^{1}$

\section{TECHNOLOGIES FOR CHEMICAL AND BIOLOGICAL COUNTERTERRORISM}

The LLNL CBNP is organized with four program areas: Instrumentation and Knowledge Management, Systems and Deployments, Applied Biodefense Sciences, and Law Enforcement and Intelligence. ${ }^{2}$ Also, we have key activities focused on Informatics, Forensic Sciences and Chemistry and Materials Science, Modeling and Prediction, Restoration Strategies and Technologies. Our management philosophy is similar to that found in venture capital - we use market pull from end-users and stakeholders to prioritize our science and technology (S\&T) investment strategies and we use basic and applied S\&T to inform stakeholders of what is possible. Early successes of the LLNL CBNP were achieved by leveraging several ongoing programs in national security, including nonproliferation of weapons of mass destruction. In the areas of biology and biotechnology, LLNL played a key role in starting and implementing the Human Genome Project and, more recently, the Microbial Genome Program. As a result of such efforts, we have resident expertise and an established infrastructure in high-throughput discovery sciences and the field of bioinformatics. LLNL also has a medical technology program that works closely with industry and university medical centers to accelerate translation of new 


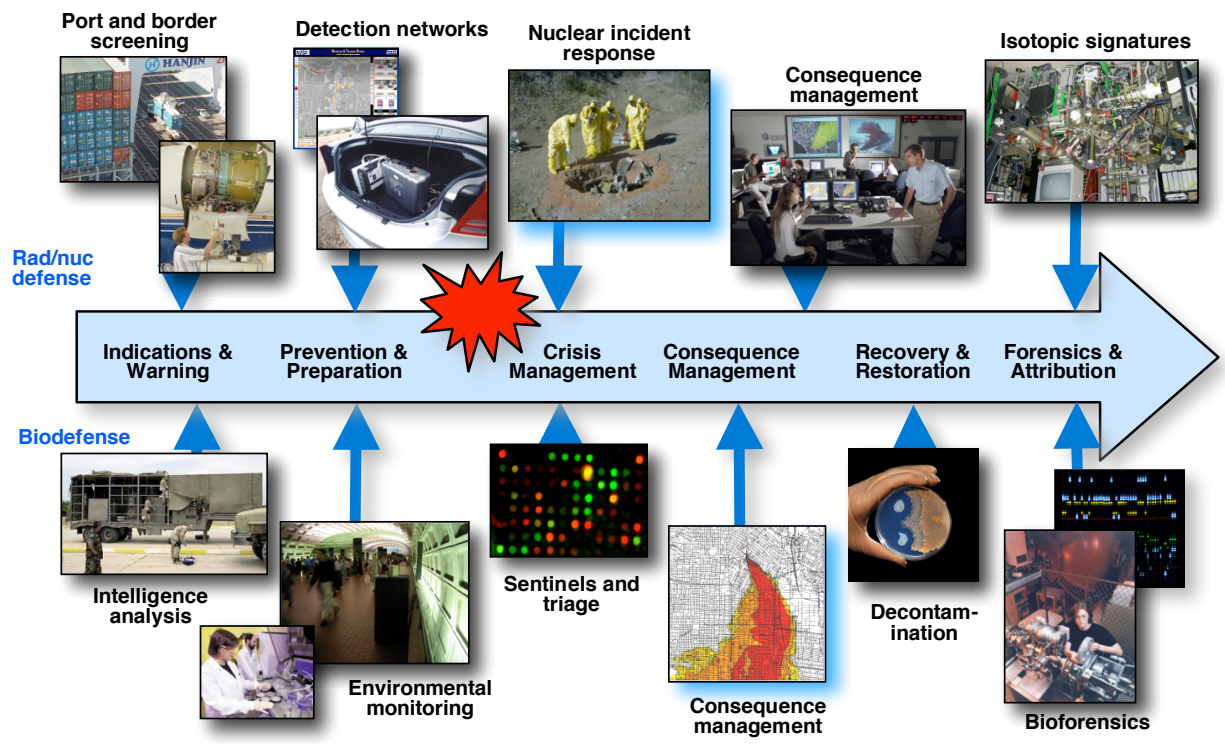

Fig.1. LLNL is applying advanced science and technology to all elements of an end-to-end defense against WMD terrorism.

technologies into clinical systems. Because of this heritage, our program's culture focuses science and technology on national security challenges with aggressive engagement of key stakeholders and industry. An important part of the science-based culture is the use of peer review to help define requirements, conduct reviews at the scientific and program levels, improve our understanding of evolving issues, and communicate new findings. ${ }^{3}$

Our early focus on biodetection followed an assessment of the challenges and gaps associated with counterbioterrorism. Because medical interventions are more effective when administered soon after or even before exposure to a pathogen, great value can be derived by detecting exposure as early as possible. We initiated a development process to put a series of systems with new capabilities into the field - systems with a focus on an environmental monitoring concept called "detect to treat." Anticipating the importance of commercialization to make our systems and technologies broadly available, we also implemented the practices and priorities needed to work with industry. Our commitment to placing systems in the field was evident even in the first project - a prototype flow-cytometry based biodetection system. After less than two years from project start, the prototype was taken to the U.S. Army Dugway Proving Grounds in Utah for blinddetection challenges. In parallel, LLNL developed a rapid and miniaturized technology for nucleic acid detection based on the polymerase chain reaction (PCR). ${ }^{4}$ The PCR technology was licensed to the start-up company Cepheid. Commercial implementation of this technology returned to LLNL when we employed the Cepheid Smartcycler ${ }^{\mathrm{TM}}$ in a field laboratory that supported the 2002 Winter Olympics in Salt Lake City, Utah, as part of the Biological Aerosol Sentry and Information System (BASIS). ${ }^{5}$

Participation of key stakeholders, including law enforcement and public health officials, during the development process was critical to the success of BASIS. In 2003, BASIS was adopted as the architecture for the national BioWatch network. Our program has analyzed roughly a half million BASIS-like samples for pathogens since October 2001. To improve performance, we have developed multiplexed and high-throughput technologies. To reduce the cost of BASIS-like systems (the most expensive component is personnel), we have developed and are field-testing the Autonomous Pathogen Detection System (APDS) that automatically collects and processes aerosol samples. ${ }^{6,7}$ Identification is done with an antibody sandwich multiplex assay (up to 100 simultaneous tests), followed by nucleic acid detection with flow-through PCR. Together with the Centers for Disease Control and Prevention (CDC), our pathogen signatures have been developed into assays for the Laboratory Response Network (LRN). Our partnership with the CDC greatly enhances the utility of our systems in public health. Looking to the future of detection and identification, we are investigating host-based signatures that can provide earlier detection of infection and determination of risk. Figure 2 shows examples of how LLNL's biosecurity work has been applied over the past few years. 


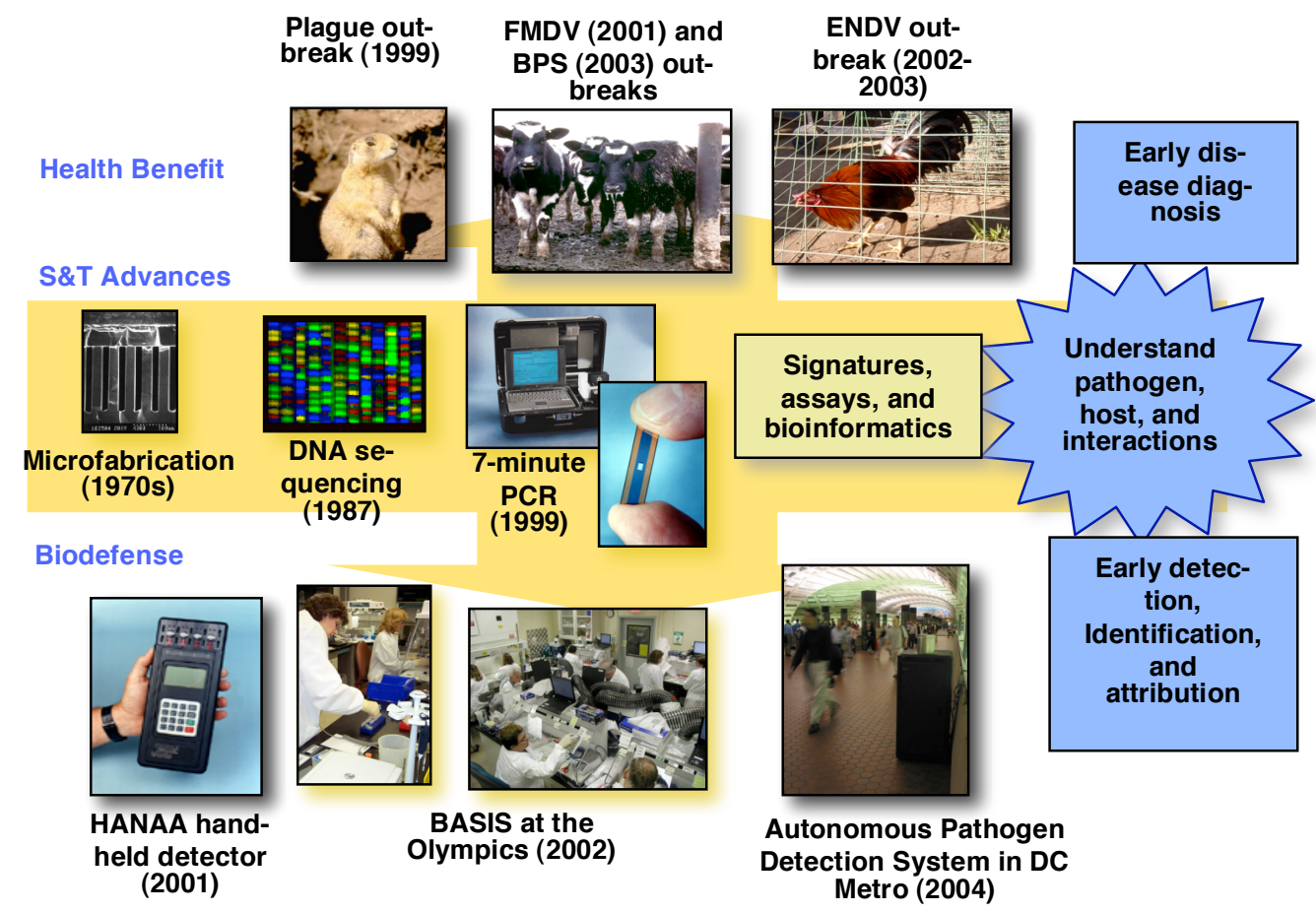

Fig. 2. LLNL has developed environmental monitoring systems that provide rapid detection with low false alarm rates. Development at application of these systems have been driven by recent real-world events such as the outbreaks of plague, foot and mouth disease virus (FMDV), bovine papular stomatitis (BPS), and Exotic Newcastle disease (ENDV) in poultry.

We also are developing a fast-response (less than a second) aerosol mass spectrometer that analyzes single particles and may have several applications, including "detect to warn" and medical and contamination triage. Commercial systems exist that can identify airborne pathogenic spores, but they take days or, at best, hours to produce results. LLNL's bioaerosol mass spectrometry (BAMS) system ${ }^{8}$ can successfully distinguish between two related but very different anthrax spore species. It can also sort out a single spore from thousands of other particles - both biological and nonbiological - with no false positives. The biomedical aspects of this work are funded by Laboratory Directed Research and Development (LDRD) at LLNL and the biodefense aspects are funded by the Technical Support Working Group and Defense Advanced Research Project Agency of the Department of Defense.

Earlier detection was not the only capability gap identified in our assessment. The U.S. faces shortfalls in its ability to prevent, prepare for, and respond to chemical or biological terrorism. In the area of prevention, we are investigating new "signatures" associated with biological and chemical warfare agents. An example of a signature for a biological agent is a specific sequence of DNA or certain proteins. Because many biological agents occur naturally, we must be able to discriminate between terrorist activities involving an agent and naturally occurring background levels of that agent. Thus, we are working to establish baseline observations of background and near-neighbor signatures.

Preparing for terrorist use of biological or chemical agents requires diligent planning. We contribute to the planning process through exercises designed to evaluate the systems, tools, and technologies intended for response. Such exercises yield valuable information for other aspects of readiness, such as sensor networks, conduct of operations, and improved placement of interventions. Because our biology and signature discovery projects produce potential leads for medical countermeasures, we are beginning to explore mechanisms and partners that can bridge the gap between promising agent-specific binding discoveries and pharmaceutical development.

In the area of response, we participate with stakeholders to develop improved tools, architectures, and concepts of operation. Our foci are restoration of operations (including rapid decontamination), forensics and attribution, and fate and transport for decision support. An example is atmospheric prediction of agent dispersion, described below, provided in minutes to decision-makers and using local up-to-date meteorological data. 
Our Forensic Science Center (FSC) is one of two U.S. laboratories certified to receive and process samples under the Chemical Weapon Convention. The FSC can quickly respond to government sponsors during developing situations where the latest technologies are needed to solve extraordinary problems. The FSC is staffed with scientists and other technical experts whose training, background, and experience include both discipline expertise and unique knowledge about WMD materials that could be utilized in a terrorist or other form of attack. The FSC is also an ISO-17025 accredited laboratory; an OPCW (Organization for the Prohibition of Chemical Weapons) designated laboratory, as well as a member of the Food Emergency Response Network (FERN). The FSC has set up Memoranda of Understanding (MOUs) with the FBI and FDA and is interacting with Customs and Border Patrol (CPB), USDA, and Secret Service.

Even though we have made substantial progress since 2001, reducing the time to intervene following a terrorist event remains a top priority. Thus, in addition to our original strategy based on gap analysis, we have two new foci: (1) science-based threat and vulnerability characterization, and (2) preparation for a large-scale event with an unexpected agent. With these two themes in mind, we are integrating projects from the gap analysis, incorporating knowledge about a threat, and preparing for the next great challenge to devise systems that can be integrated into public health and scaled to meet emerging and engineered threats. Our intent is to focus on key barriers to preparedness and, therefore, the issues with the greatest impact on successful counterterrorism response to biological and chemical warfare agents. We are aligning our internal LLNL investments to these themes and are looking for partners who share this vision. The Biodefense Knowledge Center is an outstanding example that we hope to build upon.

Out goal for environmental monitoring is to develop effective systems with low false alarm rates. Together with Los Alamos National Laboratory (LANL), LLNL developed the BASIS architecture for environmental monitoring of special events, like the Salt Lake City Olympics. LLNL proposed and implemented a new strategy for nucleic acid signature discovery that utilizes a computer-based screening and high-throughput experimental testing. The signature discovery strategy has been utilized in support of BASIS as well as the CDC Laboratory Response Network. The LLNL field deployable laboratory has been used for BASIS, National Special Security Events, and BioWatch. LLNL has also developed a next-generation environmental monitoring system - the Autonomous Pathogen Detection System or APDS.

The restoration and response goal is to shorten the time to effectively intervene. LLNL's National Atmospheric Response Advisory Capability (NARAC) provides tools and services that map the probable spread of hazardous material accidentally or intentionally released into the atmosphere. ${ }^{9}$ NARAC provides atmospheric plume predictions in time for an emergency manager to decide if taking protective action is necessary to protect the health and safety of people in affected areas. Shown in Figure 3 are plume plots of the atmospheric dispersion caused by the petroleum barge fire at Staten Island on February 21, 2003. LLNL had placed a prototype of the LINC system in New York just a few weeks before. LINC (Local Integration of NARAC in Cities) is in use in four other cities and its use is expanding.

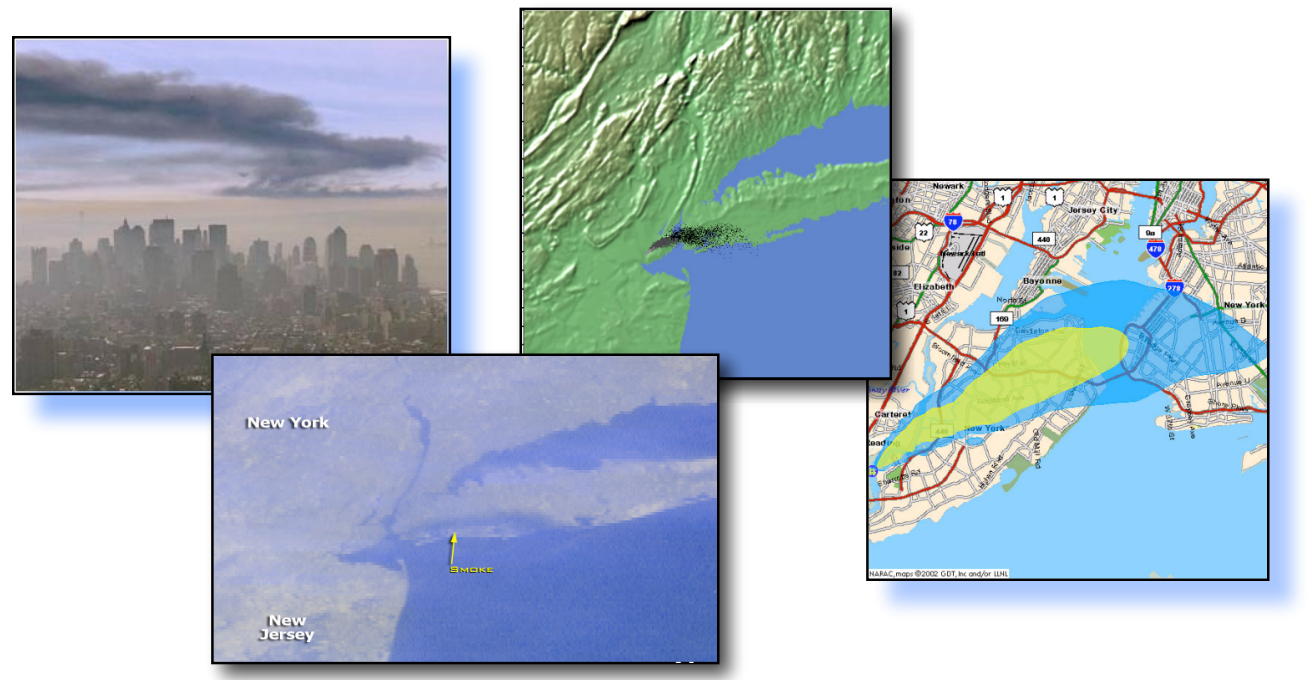

Fig. 3. Real-time weather data combined with CBRNE source information and sensor data is used to provide 3D plume models and predictions of population and facility exposure and human health effects. This information can be used to develop response strategies and prove recommendations for protective action. 


\section{TECHNOLOGIES FOR RADIOLOGICAL AND NUCLEAR COUNTERTERRORISM}

The newly appreciated challenge of detecting nuclear and radiological threats tests our ability to detect and characterize small radioactive signatures in a world with a comparatively large radioactive background. Much of the challenge in developing sensitive detection systems involves making them suitable for real-world applications - portable, rugged, battery-powered, lightweight, inexpensive, and easy to use by non-experts. The design of such systems is made possible by advances (the result of decades' worth of prior work at LLNL and elsewhere), in areas such as detection materials, microfabrication, microprocessors, data processing algorithms, and electromechanical cooling.

LLNL has been developing radiation-related technologies for decades, and continues to adapt radiation detection devices for national needs, serving a broad distribution of agencies, including DHS, DOE, the Defense Threat Reduction Agency (DTRA), the Defense Advanced Research Projects Agency (DARPA), the National Institutes of Health, the California Breast Cancer Research Program, and the National Aeronautics and Space Administration (NASA). Highlight achievements include the development of:

- A mechanically cooled germanium detector that is capable of providing gold-standard spectroscopic inspection in a hand-held device (now marketed by Ortec as the Detective)

- The Gamma-Ray Imaging Spectrometer (GRIS), which is a coded aperture gamma-ray imaging system that was developed for use in treaty inspections to nonintrusively monitor the location of nuclear missile warheads.

- The Fission Meter, which uses the unique property of multiplying quantities of special nuclear material (SNM), increased variance of neutron emissions compared to a random Poisson distribution, to sensitively detect significant SNM quantities

Current detection development efforts focus on securing cargo, enabling widespread, worldwide detection, transforming remote and mobile detection, and developing new radiation detection materials. These efforts are informed by LLNL participation in nuclear threat assessment and reachback, operational detection-system testbeds, and interdicted-material forensic analysis and attribution.

Each year, some 48 million cargo containers move between the world's ports. More than 6 million of these enter the U.S., but only about 2 percent are opened and inspected when they arrive at U.S. seaports. To secure cargo, we are developing active interrogation systems that detect unique nuclear signatures of fissile materials, including delayed gamma-ray emissions following fast neutron interrogation, correlated neutron signatures, initiated by either cosmic rays or $60 \mathrm{keV}$ neutron interrogation, and nuclear fluorescence, initiated by high-energy x-ray interrogation. ${ }^{10}$ Figure 4 shows a system currently under development that uses $7 \mathrm{MeV}$ neutrons to interrogate cargo, resulting in a delayed gamma-ray signature that is characteristic of highly enriched uranium.
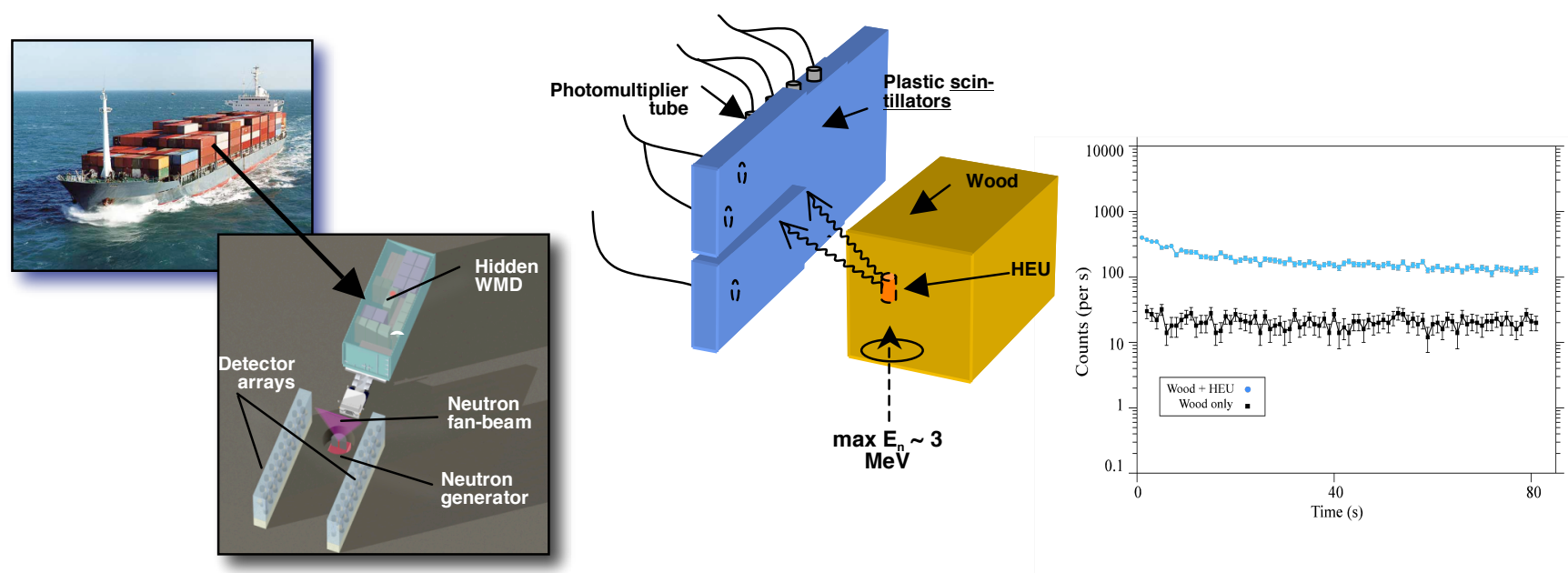

Fig. 4. Active neutron interrogation of cargo containers uses lower energy interrogating neutrons (3-7 MeV vs. $14 \mathrm{MeV}$ ), which eliminates the nitrogen interference due to oxygen activation. In the graph on the lower right, the signal due to a small amount of highly enriched uranium (HEU) in a wooden enclosure is observed to be 5-10x above background. 
To enable widespread, worldwide detection, LLNL investigators are developing low-cost, spectroscopic detection systems, along with algorithms and information management concepts to make widespread detection feasible and insightful. For example, we are developing a handheld cell-phone-based radiation detector called RadNet (Figure 5). ${ }^{11}$ RadNet uses a low-power, pixelated cadmium-zinc-telluride detector to provide moderate-resolution gamma spectroscopy at low cost. Standing alone, each unit serves as a radiation detector and identifier. By exploiting the cell phone's GPS capabilities, multiple RadNet units can be deployed as a detector network, communicating with a "base station" to monitor a wide area and, in the event of a detection, track the movement of nuclear materials.

We are developing technology to transform remote and mobile detection by harnessing gamma-ray imaging concepts envisioned initially for astrophysics and medical applications to capture the spatial signature of radiological or fissile materials to significantly enhance detection range and background discrimination. We are pursuing two complementary approaches to gamma-ray imaging: near-term development of a scintillator-based, coded-aperture imager for wide-area imaging and longerterm development of a compact Compton imager, which promises substantially greater sensitivity and lower weight than collimator-based imaging systems.

Finally, we are developing new scintillator and semiconductor radiation detection materials to provide high-resolution spectroscopy at room temperature. These materials impact all areas of radiation detection, and would dramatically enhance system performance and reduce the large procurement costs that drive national deployment. LLNL scientists are currently developing transparent ceramics, sintered from nanocrystalline particles, as high-resolution scintillators and aluminum antimonide as room-temperature, semiconductors for robust, ubiquitous use as gammaray detectors.

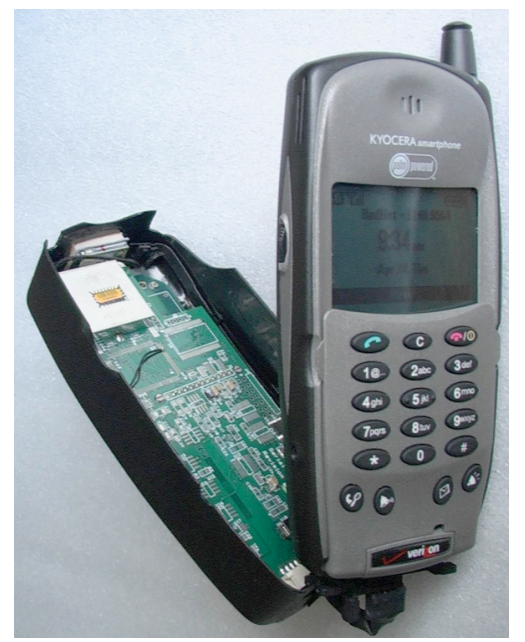

Fig. 5. The RadNet handheld cell-phone-based radiation system uses a low-power, pixilated cadmium-zinc-telluride detector to provide gamma-ray spectroscopy at low cost.

\section{TECHNOLOGIES FOR EXPLOSIVES DETECTION}

In addition to chemical wipe techniques utilizing solid phase microextraction for detection of explosive residue, LLNL is exploring a variety of different explosive detection technologies. Drawing on our expertise in x-ray and neutron detection, two different techniques are being developed for imaging explosives or other materials of interest. The first technique augments traditional transmissive $\mathrm{x}$-ray imaging, typically found in standard airport $\mathrm{x}$-ray imaging systems, with $\mathrm{x}$-ray backscatter imaging that is more sensitive to the composition, density, and atomic number of the materials being interrogated. This technique, shown in Figure 6 is still under development and requires trained technical operators to properly analyze and interpret images. Pulsed fast neutron analysis is another technique that can identify materials of interest based on neutron capture gamma ray emissions that are specific to particular atoms. This technology requires significant R\&D to improve false alarm rates prior to being a practical solution for explosives detection in containerized cargo. $^{12,13}$

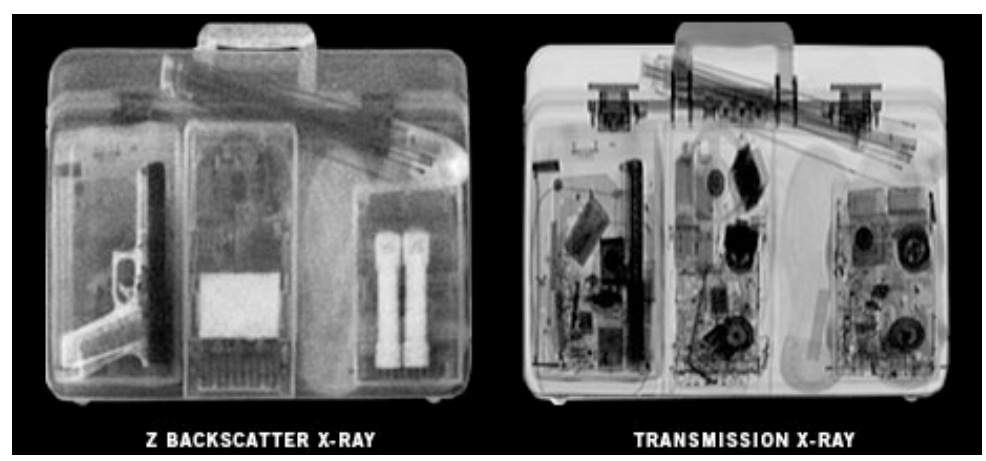

Fig. 6. In the images above the same luggage is imaged using transmission x-ray radiography (right) and backscatter imaging (left). The presence of a pistol and other materials with similar characteristics are obvious in the backscatter image, while being nearly unobservable in the transmission $\mathrm{x}$-ray. 


\section{PERSISTENT SURVEILLANCE}

LLNL has developed a very wide field of view moving imagery system called Sonoma, which was originally designed for nonproliferation and tactical military applications. ${ }^{14-16}$ The goal of Sonoma is to identify and track up to 10,000 moving objects in real time over large areas, up to the size of a small city. With this capability a user can establish vehicle movement patterns both spatially and over time, thus establishing connections between both known and unknown objects or events of interest, the time history of those connections, and identifying new objects or events. The information provided by this system can be used for tracking facility activity levels, traffic at choke points, and establishing connections between known facilities and other unknown facilities. Applications include real-time traffic monitoring, special event monitoring, border patrol, and maritime domain awareness.

The system can also cue other sensors to further investigate a target of interest or to respond to cues from other sensors or intelligence sources to locate and track them. Real-time processing capability enables the user to act on the imagery provided by the system in a timely manner. In addition, because all the imagery taken with is archived, along with all metadata needed to properly geo-rectify the imagery, the data can be used for forensic analysis following an event.

Initial demonstrations of the Sonoma capability, a number of experiments were performed in collaboration with the U.S. Border Patrol. The system demonstrated the capability to see the movement of vehicles with a resolution of about 1 meter, and even the movement of people at higher resolution. Deploying Sonoma systems along borders would offer realtime surveillance of traffic without the need for manned patrols. Furthermore, airborne deployment provides constant surveillance in multiple directions, improving the chance of catching border transgressions.

The Sonoma sensor uses commercially available cameras, lenses, and simple mounting hardware together with a mosaic optical stitching technique to provide a large field of view (FOV). All cameras share a common aperture and are focused to infinity, enabling rapid replacement in the field without calibration. The resolution of the imagery can be changed either by changing the altitude of the airborne platform or by changing out the bayonet-mounted optics for different focal length lenses. This technique is scalable to large aperture arrays in both the visible and infrared spectrum.

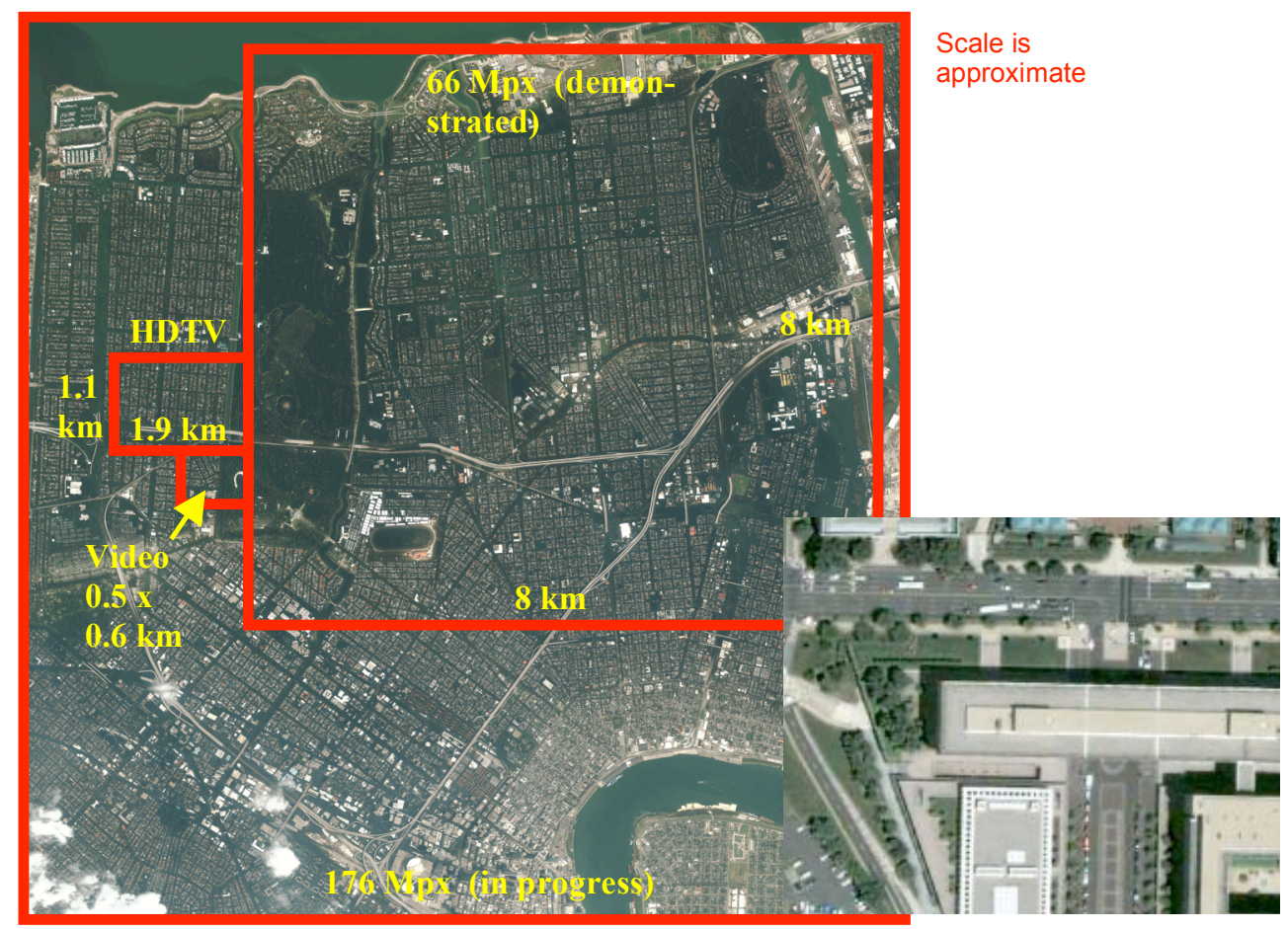

Fig. 7. Sonoma high-resolution, broad field-of-view coverage superimposed on recent satellite image of New Orleans flooding in the aftermath of Hurricane Katrina gives an example of the areal coverage associated with current and future sensor systems. The red squares show different fields of view for a given altitude provided by standard video, HDTV video, and different generations of Sonoma sensor systems. The inset on the lower left shows the approximate quality of the imagery anywhere in the full image data. 
Sonoma's current camera system is a 66 Megapixel (Mpx) system that is capable of continuously monitoring a userselected area in a field of view as great as $8 \mathrm{~km} \times 8 \mathrm{~km}$, from an airborne platform at 10,000 to 20,000 feet above ground level. A new $176 \mathrm{Mpx}$ 16-camera system is currently being designed and should be available later this year that would provide area coverage of up to about $12 \mathrm{~km} \times 12 \mathrm{~km}$. Figure 7 shows comparisons of standard video capabilities with different generations of the Sonoma sensor system. For example a standard CCD camera can provide coverage over 0.5 $\mathrm{km} \times 0.6 \mathrm{~km}$. High definition TV (HDTV) improves the FOV by roughly a factor of two but neither camera can image the area of a city under these conditions. The Sonoma system has adequate spatial resolution $-0.8-\mathrm{m}$ ground sampling distance (GSD) - and temporal resolution - 2-Hz frame rate - to track all moving targets of interest with low tasking latency. On-board algorithms, implemented in graphics processors, enable real-time data processing and display. A communications link to a ground station has been demonstrated using a two-camera, $22 \mathrm{Mpx}$ system to transmit data in real time with automated tracking algorithms. However, current RF communication systems are not adequate for real time down-linking of larger camera system data without the use compression techniques that are currently under development at LLNL and elsewhere.

The key to Sonoma's real-time image processing is specialized software executed using standard graphic processors to enable a 100-times acceleration of the georegistration algorithms, so that the imagery from each camera in the mosaic can be processed in real-time. Sonoma sensors are mounted with a co-boresighted Global Positioning System/Inertial management Unit (GPS/IMU) on a high-precision gimbal. The gimbal is locked on the center of the field of regard to $<10 \mathrm{mrad}$; as the platform moves, the center of the image remains stationary. The geospatial coordinates and elevation data are recorded directly into the header of each frame of camera data, enabling the georegistration and geolocation of each pixel in every frame. Using this information, data can be georegistered in real time to a flat-earth model and reprojected to a nadir viewing perspective. The motion of the platform is effectively removed, making the sensor system platform independent. The net result is that the background is frozen, and all moving objects are easily identified.

The ability to "freeze" the background of an $8 \mathrm{~km} \times 8 \mathrm{~km}$ area from an aerial platform and to highlight just the moving objects in the scene allows the full-resolution imagery associated within a selectable region of the moving object to be saved and transmitted to the ground at $2 \mathrm{~Hz}$. These data squares can then be superimposed on a full-resolution contextual image sent down less frequently, typically once per minute. The resulting reduction in data volume transmitted to the ground is equivalent to approximately six orders of magnitude compression. Automated tracking algorithms that highlight each moving object can then be applied to the resultant imagery.

The Sonoma system has been demonstrated in a series of four flight experiments conducted over the past two years on three different platforms, including a helicopter and two different fixed wing aircraft. These experiments have generated 10 terabytes of broad-area moving imagery over four different geographical locations. For each data set taken, a coordinated set of ground scenarios (with vehicles and/or people) was executed to evaluate the effectiveness of the Sonoma system. These tests demonstrate all of the key components of the system in action simultaneously, including (a) onboard, real time data processing, (b) high-bandwidth communications, and (c) a ground station with specialized processing to reconstruct the imagery in real time sensor suite.

In order to maintain persistent coverage over a defined region, Sonoma's gimbal is locked pointing to a specific coordinate, and the airborne platform flies either in a circle or figure eight about that point. Because the Sonoma data is georegistered in real time, it can be mapped to a digital terrain map to provide elevation data. However, in some cases, accurate maps of an area do not exist. Because Sonoma obtains imagery from several perspectives as the platform circles, it can provide the data necessary to create a digital terrain map of an area in real time. Such a capability would be invaluable for situational awareness or for assessing topographical changes in the aftermath of an environmental or terrorist event.

\section{CONCLUSION}

LLNL's mission includes performing research and development that supports nonproliferation of weapons of mass destruction. This work has led to a major focus on developing technologies for counter-terrorism, supported by a number of U.S. government agencies. The work described in this paper is necessarily only a small part of the actual work taking place at LLNL, other laboratories, universities, and in industry. LLNL's role in providing technology is clearly focused on science-based R\&D leading to innovative detectors that are integrated into operational systems. LLNL's focus on the 
development cycle has led to a venture-capital-style management approach that depends on open communication with and participation of end-users and stakeholders. It also motivates innovation-looking to the S\&T base for strategies that produce revolutionary capabilities for end-users. Just like the venture investor, our management goal is to provide value to the end user by managing the transitions from S\&T, to proof of principle, to demonstration and pilots, to operational systems. Our program benefits from participating in all of these stages, from basic research through operational support. We leverage that experience and the network of end-users and stakeholders to set priorities as we conduct the long-term visionary $R \& D$ required to make the science and technology breakthroughs that will make it possible to meet future needs and address new threats.

\section{ACKNOWLEDGEMENTS}

The authors are indebted to the many dedicated researchers that are contributing daily to the nonproliferation and counterterrorism work described in this paper. This work has been performed under the auspices of the U.S. Department of Energy by the University of California Lawrence Livermore National Laboratory under Contract No. W-7405-Eng-48.

\section{REFERENCES}

1. "Key Challenges in Homeland Security," Lawrence Livermore National Laboratory, UCRL-BR-151001 (2004).

2. "Biological and Chemical Security," Lawrence Livermore National Laboratory, UCRL-TR-205698, August 2004.

3. J. P. Fitch, E. Raber, and D. R. Imbro, "Technology Challenges in Responding to Biological or Chemical Attacks in the Civilian Sector," Science 302, 1277-1460 (2003).

4. P. Belgrader, W. Benett, D. Hadley, J. Richards, P. Stratton, R. Mariella, Jr., and F. Milanovich, "PCR Detection of Bacteria in Seven Minutes," Science 284, 449-450 (1999).

5. D. Imbro, A. Heller, "BASIS Counters Airborne Terrorism," Science and Technology Review, UCRL-52000-03-10, Lawrence Livermore National Laboratory, 6-7, October 2003.

6. M. T. McBride, D. Masquelier, B. J. Hindson, A. J. Makarewicz, S. Brown, K. Burris, T. Metz, R. G. Langlois, K. W. Tsang, R. Bryan, D. A. Anderson, K. S. Venkateswaran, F. P. Milanovich, and B. W. Colston, Jr., "Autonomous Detection of Aerosolized Bacillus anthracis and Yersinia pestis," Analytical Chemistry 75 (20), 5293-5299 (2003).

7. R. Langlois, A. Parker, "Detecting Bioaerosols: When Time is of the Essence," Science and Technology Review, UCRL-52000-04-10, Lawrence Livermore National Laboratory, 4-6, October 2004.

8. V. Riot, G. Rennie, "Identifying Airborne Pathogens in Time to Respond, Science and Technology Review, UCRL52000-05-10, Lawrence Livermore National Laboratory, 8-9, October 2005.

9. J. S. Nasstrom, G. Sugiyama, R. Baskett, S. Larsen, and M. Bradley. "The National Atmospheric Release Advisory Center (NARAC) Modeling and Decision Support System for Radiological and Nuclear Emergency Preparedness and Response, UCRL-JRNL-211678, International Journal of Risk Assessment and Management Special Issue: Nuclear and Radiological Emergency Preparedness - The Role of Monitoring and modeling in an Emergency Situation, April 2005.

10. D. Slaughter, D. Sprouse, "Screening Cargo Containers to Remove a Terrorist Threat," Science and Technology Review, UCRL-52000-04-05, Lawrence Livermore National Laboratory, 12-15, May 2004.

11. S. Labov, G. Rennie, 'Radiation Detection on the Front Lines," Science and Technology Review, UCRL-52000-0409, Lawrence Livermore National Laboratory, 4-11, September 2004.

12. Detection of Explosives for Commercial Aviation Security, National Research Council. Washington, D.C., National Academy Press. National Research Council. 1993.

13. Second Interim Report of the Committee on Commercial Aviation Security. Washington, D.C., National Academy Press. 1997.

14. L. Flath, "Using Commercial Graphics Processors for Real Time Geo-registration,” UCRL-PRES-200957, November 2003.

15. L. Flath, M. Kartz and R. Frank, "Utilizing Commercial Graphics Processors in the Real-Time Geo-registration of Streaming High Resolution Imagery," UCRL-PRES-205737, August 2004.

16. M. Kartz, D. Pennington, L. Flath, C. Brown, J. Marion, S. Reuther, A. Vanderbilt, D. Clark, and A. Skene, "Sonoma Persistent Surveillance Program: An Experimental Assessment of Object Tracking Requirements," UCRLTR-208865, August 2005. 